\section{Copper, zinc and magnesium plasma levels in epilepsy}

Sir: Copper and zinc are known to produce seizures in animals at low dosage, this being possibly related to the inhibition of $\mathrm{Na}-\mathrm{K}$ ATPase activity. ${ }^{1}$ Magnesium alters calcium mobilistaion and may stabilise excitable membranes. Theoretically, trace metals may have a role to play in the production of seizures and their control in humans. This concept has led to several studies the results of which are conflicting. Barbeau and Donaldson ${ }^{1}$ demonstrated a reduction in zinc levels in epileptic patients but this was not confirmed by Vasiliades and Sahawneh. ${ }^{2}$ Schott and Delves ${ }^{3}$ reviewed a small number of epileptic patients and found normal zinc but elevated plasma copper levels. Olatunbosum $e t a l^{4}$ recorded a fall in zinc and increase in serum copper. Christiansen et al ${ }^{5}$ observed a significant decrease in serum magnesium which was not confirmed by Katz et $a l .{ }^{6}$ Such conflicting results suggested the need to assess our own patients, the aim being primarily to determine initially whether zinc, copper and magnesium differed between epileptics on anticonvulsant therapy and normal healthy volunteers and, secondly, within the epileptic population whether there was any correlation between these metal levels, therapy and seizure frequency.

Forty-nine patients from our Out-patient Clinic were randomly selected. All were on anticonvulsant therapy and had a diagnosis of primary generalised epilepsy, partial complex epilepsy or partial complex epilepsy with secondary generalisation. The population did not include patients with known intracranial pathology and no patients were on other regular medication. Twenty-five healthy volunteers were matched for age, height and weight. Blood was taken for electrolytes, liver function tests and full blood count in addition to plasma copper, zinc and serum magnesium, the latter being corrected for albumin. The epileptic group had serial estimations of their anticonvulsants in order to achieve therapeutic levels. $64 \%$ of our epileptics had plasma anticonvulsant levels within the therapeutic range, $32 \%$ were subtherapeutic largely owing to poor drug compliance and $4 \%$ were in the toxic range, though only one patient had symptoms.

To assess reproducibility of trace metal estimations these were serially analysed. Specimens were taken between 0.9 .00 and 11.00 am to obviate any diurnal variation. Copper, zinc and magnesium were analysed by atomic absorption spectrophotometry and anticonvulsant estimations by the enzyme immunoassay method EMIT. Standard statistical methods were used and comparisons made using Student's $t$ test and analysis of variance.

Table Plasma Copper, Zinc and Serum Magnesium levels (Mean \pm ISD) in patients with Epilepsy, in control objects

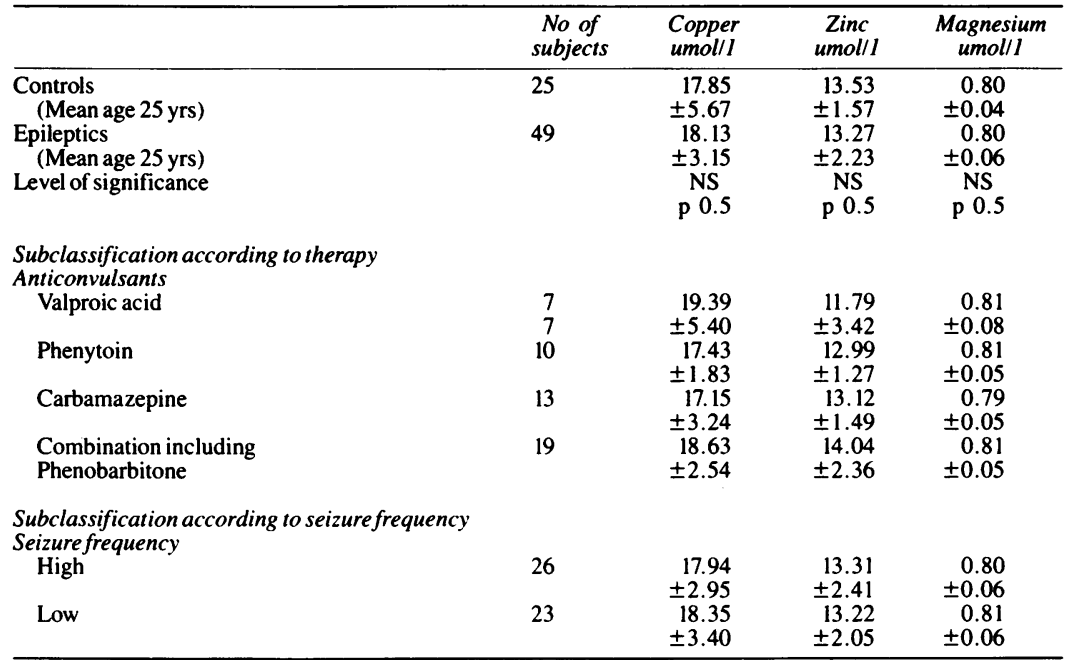

All values from epileptic groups show no statistically significant difference from controls.

From the tables it can be seen that there was no difference in plasma copper, zinc and serum magnesium between the epileptic group and the healthy volunteer controls. Likewise when the epileptic groups were subclassified according to their anticonvulsant therapy, the group treated with valproic acid had a higher copper and lower zinc but this difference was not found to be statistically significant. Phenytoin, carbamazepine and phenobarbitone also had no effect on blood metal levels. The epileptic group were arbitrarily divided into high and low seizure frequency; high frequency being more than one seizure per month and low frequency being less than one seizure per month. There was no difference between these groups. The epilepsy group was again subdivided according to the international classification of primary generalised, focal and secondary generalised epilepsy. This was based on clinical and EEG evidence. Again there was no difference between the groups.

WGJ SMITH
I BONE
Stobhill General Hospital,
and Institute of Neurological Sciences,
Southern General Hospital
Glasgow, G5I 4TF, UK

\section{References}

' Barbeau A, Donaldson J. Arch. Neurol. 1974: 30:52-8.

${ }^{2}$ Vasiliades J, Sahawneh T. Clin. Chem. 1975: 21:637-8.

${ }^{3}$ Schott G, Delves H. Br. J. Clin. Pharmacol. 1978: 5(3):279-80.
${ }^{4}$ Olatunbosum D, Akindele M, Adeniyi F, Bademosi O. Niger Med. J. 1978;8(2):124-5.

${ }^{5}$ Christiansen C, Nielson S. Bri. Med. J. 1974:1:198.

${ }^{6}$ Katz S, Gerstman I, Lautenbacher H, Hediger M. Br. Med. J. 1976;1:341.

\section{A penetrating orbitocranial injury presenting as a gaze palsy}

Sir: When examining a patient who has been stabbed in the region of the eye it can be difficult to assess clinically how deeply the orbit has been penetrated. The external wound is often relatively insignificant and the eye frequently escapes injury, being pushed aside into the orbital fat. ${ }^{1}$ Radiological examination can be unhelpful. ${ }^{2}$ The patient may develop a neurological deficit resulting from intracranial haemorrhage or infection before it becomes evident that an apparently trivial injury has in fact penetrated the cranial cavity.' A high proportion of transorbital stab injuries therefore go undetected initially and death frequently occurs from intracranial complications. $^{1-9}$ A penetrating orbitocranial injury was recently seen in which an important neurological localising sign at the initial examination gave forewarning that the damage was much more extensive than at first supposed.

A 22-year-old drama student was struck above the right eye by a fencing foil (figure). On his arrival in the Accident and Emergency Department the right eye was 




Figure (a) The fencing foil responsible for the injury. (b) Enlarged view of the tip of the foil.

blind though the globe was intact and the skin of the upper eyelid unbroken. Proptosis was noted but there was no evidence of pulsation and a bruit could not be detected. The right pupil exhibited complete afferent and efferent defects in the response to light and failed to constrict on accommodation. The presence in addition of right-sided ptosis, total external ophthalmoplegia, and corneal anaesthesia suggested a diagnosis of traumatic orbital apex syndrome ${ }^{10}$ on the basis of examination of the right eye alone. Fundus examination revealed no defect of perfusion of the right optic nerve head. Examination of the left eye at this stage produced evidence of intracranial penetration. The visual acuity was normal and the pupil reacted briskly to light and accommodation. The left eye could not be adducted beyond the midline when the stimulus was command or pursuit, optokinetic, or vestibular but adduction occurred on attempted convergence. Although the complete external ophthalmoplegia affecting the right eye prevented full testing of conjugate movements, these findings were considered indicative of a horizontal gaze palsy resulting from damage to the supranuclear horizontal gaze centre in the pons or to the adjacent longitudinal bundle. The left fundus was normal. The patient was admitted to hospital. Neurological examination at this stage demonstrated no abnormality other than those already noted. Radiological examination of the skull, orbits, and optic foramine was normal and in particular there was no evidence of damage in the region of the right superior orbital fissure. A CT scan showed the proptosis as the only abnormality. Neurological observations were begun. Within 2 hours of admission there developed a left-sided hemiplegia and a further CT scan showed attenuation in the region of the brain stem. The absence of a carotico-cavernous fistula was confirmed and the signs were considered to have resulted from oedema rather than from compression by haemorrhage. Tetanus prophylaxis was given and on conservative management with antibiotics and dexamethasone the left hemiplegia and the horizontal gaze palsy rapidly resolved After several weeks an efferent pupil response could be elicited on the right side corneal sensation was restored, and considerable improvement was seen in the external ophthalmoplegia. Loss of vision in the right eye was permanent and probably resulted from swelling of the optic nerve within the confines of the bony optic canal following contusion. ${ }^{1011}$ Within a month optic disc pallor was apparent. Permanent damage to cerebellar pathways resulted in ataxia and horizontal nystagmus which did not resolve.

It is evident that the foil entered the orbit through the upper conjunctival fornix producing very little external sign of injury. It traversed the orbit and, taking a course determined by the downward slope of the orbital roof, passed through the superior orbital fissure and on, lateral to the cavernous sinus, before coming to rest in the region of the pons and right cerebellar hemisphere. To date there are few reports of hemiplegia following transorbital penetrating injury, ${ }^{31213}$ and so far as is known the finding of a gaze palsy in such an injury has not previously been reported. The possibility of intracranial extension must always be considered in cases of orbital injury. It is wise to admit all potential transorbital injuries to hospital for observation' and the importance of a careful neuro-ophthalmological assessment at the initial examination cannot be overstated. ${ }^{2}$

JOHN HUNGERFORD University College Hospital, Gower Street, London, WCI

\section{References}

' Kjer P. Orbital and transorbital stab wounds. Arch Ophthalmol 1954;51:811-21.

${ }^{2}$ Bard LA, Jarrett WH. Intracranial complications of penetrating orbital injuries. Arch Ophthalmol 1964;71:332-43.

${ }^{3}$ Bulluck MH, Baker GS, Henderson JW. Injuries of the brain caused by penetration of the orbit. Minn Med 1959;42:1408-13.

${ }^{4}$ McClure CC, Jr, Gardner WJ. Transorbital intracranial stab wounds. Cleve Clin $Q$ 1949;16:118-25.

${ }^{5}$ Guthkelch AN. Apparently trivial wounds of the eyelids with intracranial damage. $\mathrm{Br} \mathrm{Med}$ $J$ 1960;ii:842-4.

- Duffy GP, Bhandari YS. Intracranial complications following transorbital penetrating injuries. BrJ Surg 1969;56:685-8.

${ }^{7}$ Webster JE, Schneider RC, Lofstrom JE. Observations upon management of orbitocranial wounds. J Neurosurg 1946;3:329-36.
${ }^{8}$ Schneider RC, Henderson JW. Penetrating orbital wound with intracranial complications. Arch Ophthalmol 1952;47:815.

9voboda J. Injury of orbit and base of skull with fatal termination. Ceskoslovenská oftalmologie 1948:72-4. Abstracted Ophth Lit 1948;2:422.

${ }^{10}$ Watson PG, Holt-Wilson AD. The traumatic orbital apex syndrome, its differential diagnosis and treatment. Trans Ophthalmol Soc UK 1968;88:361-74.

" Evans JP, Scheinker IM. Histologic studies of brain following head trauma: post-traumatic cerebral swelling and edema. $J$ Neurosurg 1945;2:306-14.

12 Larmande A, Descuns P, Margaillan A. Wound of the orbit with homolateral hemiplegia in a fencer. Rev Otoneuroophthalmol 1956;28: 440-1.

${ }^{13}$ Platt ES. Orbitocranial penetrating injuries. $A m$ J Ophthalmol 1954;37:758-63.

Focal dystonia in association with cerebral infarction

Sir: We wish to report the case of a womand who developed bradykinesia and rigidity $\frac{\mathcal{N}}{N}$ her left arm, and subsequently, a prष فे gressive fixed dystonic posture of her le hand in association with an infarct in the region of the right basal ganglia.

In 1974, when 43 years old, the patierg presented with a six month history of pre gressive weakness of the left hand, with difficulty in doing up buttons or manipulaf ing a fork. There was no family history of consanguinity or of neurological disease and the patient's previous health had been excellent. On examination there was a mild resting tremor of the left hand, with cogwheel rigidity at the elbow and wrist, and fine finger movements of the left hand were moderately impaired. The blood pressure was $230 / 140 \mathrm{~mm} \mathrm{Hg}$.

An initial diagnosis of Parkinson's disease was made and the hypertension treated, first with methyldopa and later propranolol. No anti-Parkinsonian therapy was given and after a year the tremor remitted, but there was a slight increase in the rigidity and bradykinesia in the left arm. The latter failed to respond to trials of levodopa, bromocryptine or anticholinergic treatment which was tried over the following two years. In 1977 the patient first developed an abnormal posture of the left hand and this gradually increased during the next three years. On examination at the end of 1981 she was normotensive and her left hand was now permanently maintained in a dystonic posture (fig $\mathrm{A}$ ). The medial three fingers were flexed at the metacarpo-phalangeal 